\title{
DERIVATION OF BURST STATISTICS FOR HIDDEN MARKOV CHANNEL MODELS
}

\author{
Cecilio Pimentel \\ Communications Research Group - CODEC \\ Department of Electronics and Systems, P.O. Box 7800 \\ Federal University of Pernambuco \\ 50711-970 - Recife - Brazil \\ E-mail : cecilio@npd.ufpe.br
}

\begin{abstract}
Resumo - Neste artigo apresentamos um método enumerativo para calcular expressões analíticas para as estatísticas de surtos de erros gerados por canais de comunicação modelados por cadeias de Markov escondidas (HMC). É demonstrado que a distribuição de peso de uma sequiência de erro pode ser descrita analiticamente a partir da distribuição do número de intervalos entre erros. $O$ estudo da descrição estatística do processo de erro tem aplicações imediatas no modelamento de canais reais e no projeto de esquemas de codificação para canais com memória.
\end{abstract}

Abstract - In this paper we present an enumerative method to derive analytic expressions for burst error statistics of hidden Markov channel (HMC) models with an arbitrary number of states. It is demonstrated that the error weight distribution can be analytically described from the distributions of intervals between errors. The study of the statistical description of the error process has immediate applications in the modeling of real channels, and in the designi of coding schemes for channels with memory.

Keywords: channels with memory, combinatorial methods, multigap distribution, burst statistics, error weight distribution.

\section{INTRODUCTION}

Two fundamental problems arise in the mathematical modeling of communication systems: The problem of channel modeling and the problem of applying the model to further system analysis. The aim of channel modeling is to obtain as simple an analytical model as possible that accurately reflects the important statistical description of the real error process. Therefore, we can use the statistics of the model to design coding schemes and to evaluate the performance of communication systems.

An HMC model is characterized by an underlying nonobservable Markov chain [1]. The discrete output symbol to the channel at the $k^{t h}$ time interval, $y_{k}$, is a function of the input symbol $x_{k}$ and the state of the Markov chain $s_{k}$. The channel is described statistically by the conditional probability $P\left(y_{k}, s_{k} \mid x_{k}, s_{k-1}\right)$. The difference between the input and output sequence to the channel is defined as the error sequence. Thus, for example, in a binary system the error sequence will be a sequence of zeros and ones with a one 108 indicating an error. The characteristics of the channel impairments, are incorporated into the model in such a manner to produce a statistically similar error sequence as produced by the real channel. This HMC model is suitable when the channel has a set of properties constant for a certain period of time, and then, change sequentially to another set of properties. This approach has some interesting features: (i) The model is quite general and flexible. For a broad class of real communication channels, the correlation structure of the error sequence can be accurately characterized by the proper choice of the model parameters. (ii) The model has a well known analytical description. When its parameters are known, the multidimensional probability of the error sequence can be calculated.

Some burst error statistics have been used as a criterion for selecting models to represent real channels. One issue of considerable importance is the investigation of the renewal nature of the error process. Renewal channels possess the property that the gap (sequences of zeros) lengths before and after an error are independently distributed. Examples of renewal models include Fritchman channels with one error state [1]. The Gilbert-Elliott channel [2], for example, is non-renewal. Renewal models have gained some popularity mainly due to their simplicity of analysis. However, it can be demonstrated that many real communication systems cannot be accurately modeled as renewal models $[1,3]$. The statistic called multigap distribution has been used as a test of non-renewalness of the error process. Despite this important property, no analytical expression for the multigap distribution of HMC models has been given in the literature so far. Another application of burst error statistics is the design and performance evaluation of coded systems over HMC models. Some statistics of interest for the encoder designer may include the probability of exactly $m$ errors occurring in a block of length $n$, the average burst length, and many others.

In this work we follow the theory of enumeration of constrained sequences described in [4] to enumerate a particular subset of error sequences generated by the HMC model. We will show that the probability of this subset can be obtained by acting on the generating series with a linear mapping extended as a homomorphism to the whole of the ring of all formal power series. As an application, we specialize these general expressions for the cases of Gilbert-Elliott and Fritchman channel models. Moreover, we will also use the generating series to find recurrence formulas, which are convenient for computation. The techniques developed here are not lim- 
ited to these particular models and can be readily applied to more general Markov models.

This work is organized in five sections. Section 2. describes the details of the generation of the error process with HMC models and its properties. In Section 3. we define the generating series in a ring of all formal power series in noncommuting indeterminates. A method for determining the burst error statistics from the generating series is introduced in this section. In Section 4. we give explicit expressions for the burst error statistics considered in this work. Finally, concluding remarks are given in Section 5. .

\section{MODEL DESCRIPTION}

Consider $\left\{S_{k}\right\}_{k=1}^{\infty}$ an $N$-state Markov chain with a finite state space $\mathcal{N}_{N}=\{0,1, \ldots, N-1\}$. Let $\mathbf{P}$ be an $N \times N$ transition probability matrix, whose $(i, j)^{t h}$ entry is $p_{i, j}$. The error sequence is generated by the HMC model as follows. At the $k^{\text {th }}$ time interval, the chain makes a transition from state $S_{k}=i$ to $S_{k+1}=j$ with probability $p_{i, j}$ and generates an output symbol $e_{k} \in \mathcal{N}_{2}$ (independent of $j$ ), with probability $b_{i, e_{h}}=P\left(E_{k}=e_{k} \mid S_{k}=i\right)$. We are assuming that the distribution of the initial state is the stationary distribution $\Pi=\left[\pi_{0}, \pi_{1}, \ldots, \pi_{N-1}\right]^{T}$ (where the superscript $[\cdot]^{T}$ indicates the transpose of a matrix).

The probability of an error sequence is calculated as follows. Define the $N \times N$ matrix $\mathbf{P}\left(e_{k}\right)$ whose $(i, j)^{t h}$ entry is $p_{i, j}\left(e_{k}\right)=P\left(E_{k}=e_{k}, S_{k+1}=j \mid S_{k}=i\right)=p_{i, j} b_{i, e_{k}}$, which is the probability that the output symbol is $e_{k}$ when the chain makes a transition from state $i$ to $j$. The probability of an error sequence of length $n, e_{n}=e_{1} e_{2} \ldots e_{n}$, has a matrix form given by:

$$
P\left(e_{n}\right)=\boldsymbol{\Pi}^{T}\left(\prod_{k=1}^{n} \mathbf{P}\left(e_{k}\right)\right) \mathbf{1},
$$

where 1 is a column matrix with all entries ones.

The Gilbert-Elliott channel [2] is a two-state Markov chain composed of a good state, state 0 , where errors occur with small probability, and a bad state, state 1 , where errors occur with higher probability, as illustrated in Figure 1. When the chain is in the good state the error bit $e_{k}$ is zero (correct) with probability $1-g$, or one (error) with probability $g$. Otherwise, when it is in a bad state, the error bit is zero with probability $1-b$, or one with probability $b$.

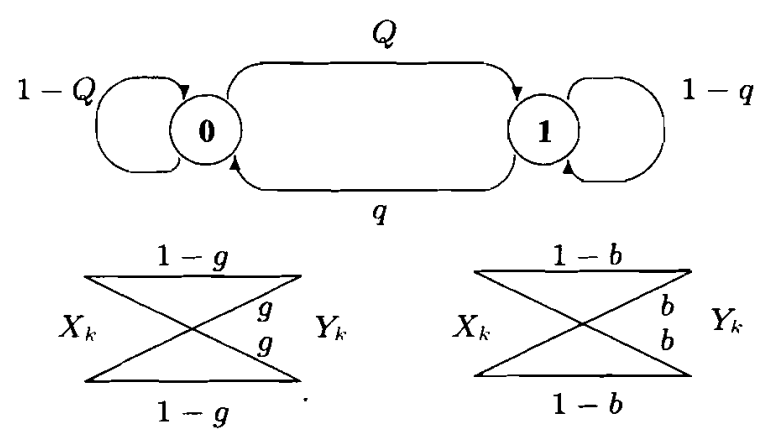

Figure 1: Gilbert-Elliott model for burst channels.
The matrices $\mathbf{P}, \mathbf{P}(0), \mathbf{P}(1)$ and $\Pi$ are given by:

$$
\begin{gathered}
\mathbf{P}=\left[\begin{array}{cc}
1-Q & Q \\
q & 1-q
\end{array}\right] \\
\mathbf{P}(0)=\left[\begin{array}{cc}
(1-Q)(1-g) & Q(1-g) \\
q(1-b) & (1-q)(1-b)
\end{array}\right] ; \\
\mathbf{P}(1)=\left[\begin{array}{cc}
(1-Q) g & Q g \\
q b & (1-q) b
\end{array}\right] ; \\
\mathbf{\Pi}=\left[\pi_{0}, \pi_{1}\right]^{T}=\left[\frac{q}{q+Q}, \frac{Q}{q+Q}\right]^{T}
\end{gathered}
$$

Some error distributions for this channel can be calculated using Equation (1). As an example, the probability the bit error is a $1, P(1) \triangleq P\left(E_{k}=1\right)$, and the probability of two consecutive ones, $P(11) \triangleq P\left(E_{k}=1, E_{k+1}=1\right)$, are given by:

$$
\begin{gathered}
P(1)=\Pi^{T} \mathbf{P}(1) \mathbf{1}=\pi_{0} g+\pi_{1} b . \\
P(11)=\boldsymbol{\Pi}^{T} \mathbf{P}(1) \mathbf{P}(1) \mathbf{1} .
\end{gathered}
$$

An HMC model can also be described as a deterministic function of a Markov chain. In the Fritchman channel, the state space $\mathcal{N}_{N}$ is partitioned into two disjoint subsets $\mathcal{A}_{0}=$ $\{0, \ldots, K-1\}$ (the good states), and $\mathcal{A}_{1}=\{K, \ldots, N-1\}$ (the bad states). The error bit generated by the model at the $k^{\text {th }}$ time interval, $E_{k}$, is a deterministic function of the current state $S_{k}$, and assumes the value $E_{k}=0$ (no error) if $S_{k} \in \mathcal{A}_{0}$ or $E_{k}=1$ (error) if $S_{k} \in \mathcal{A}_{1}$. Using the matrix $\mathbf{P}$ and this partition, we can determine the two matrices $\mathbf{P}(0)$ and $\mathbf{P}(1)$, where $\mathbf{P}=\mathbf{P}(0)+\mathbf{P}(1)$. We will consider a class of equivalent Fritchman channels (EFC) with matrix $\mathbf{P}$ having the following special structure:

$$
\mathbf{P}=\left[\begin{array}{l|l}
\boldsymbol{\Lambda}_{00} & \mathbf{P}_{01} \\
\hline \mathbf{P}_{10} & \boldsymbol{\Lambda}_{11}
\end{array}\right],
$$

where $\boldsymbol{\Lambda}_{00}$ and $\boldsymbol{\Lambda}_{11}$ are diagonal matrices. The block matrices $\mathbf{P}_{k, l}$ represent the transition probabilities from the set $\mathcal{A}_{k}$, to $\mathcal{A}_{l}$. A model with $K$ good states and $N-K$ bad states is denoted by $(K, N-K)$-EFC. In particular, the matrices $\mathbf{P}$, $\mathbf{P}(0), \mathbf{P}(1)$ and $\boldsymbol{\Pi}$ for the $(2,1)$-EFC model are given by:

$$
\begin{gathered}
\mathbf{P}=\left[\begin{array}{cc|c}
\lambda_{0} & 0 & 1-\lambda_{0} \\
0 & \lambda_{1} & 1-\lambda_{1} \\
\hline p_{2,0} & p_{2,1} & 1-p_{2,0}-p_{2,1}
\end{array}\right] ; \\
\mathbf{P}(\mathbf{0})=\left[\begin{array}{cc|c}
\lambda_{0} & 0 & 0 \\
0 & \lambda_{1} & 0 \\
\hline p_{2,0} & p_{2,1} & 0
\end{array}\right] ; \\
\mathbf{P}(\mathbf{1})=\left[\begin{array}{cc|c}
0 & 0 & 1-\lambda_{0} \\
0 & 0 & 1-\lambda_{1} \\
\hline 0 & 0 & 1-p_{2,0}-p_{2,1}
\end{array}\right]
\end{gathered}
$$




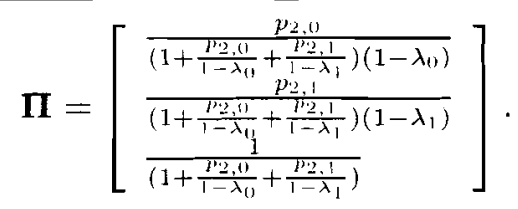

Tsai modeled a fading HF communication channel using the following (2,1)-EFC model [5]:

$$
\mathbf{P}=\left[\begin{array}{cc|c}
0.99911 & 0 & 0.00089 \\
0 & 0.73644 & 0.26356 \\
\hline 0.36258 & 0.58510 & 0.05232
\end{array}\right]
$$

\section{THE GENERATING SERIES}

The problem we will address in this section is to find the probability of error events associated with HMC models. The error events of length $n$ will be denoted generically by $\mathcal{E}_{n}$. It is well known that the probability of an error sequence is not preserved under commutation of its symbols. This prompts us to define the generating series in non-commuting indeterminates in order to keep all the information about the original sequence. So denote the generating series for $\mathcal{E}_{n}$ (error event) as:

$$
F_{\mathcal{E}_{n}}=\sum_{\boldsymbol{e}_{n} \in \mathcal{E}_{n}} x_{e_{1}} x_{e_{2}} \ldots x_{e_{n}}, \quad x_{e_{i}} \in\left\{x_{0}, x_{1}\right\}
$$

which is in $R \ll x_{0}, x_{1} \gg$, the ring of all power series in the non-commuting indeterminates $x_{0}, x_{1}$. The indeterminates $x_{0}$ and $x_{1}$ mark an error bit equal to 0 or 1 , respectively. Notice that $P\left(\mathcal{E}_{n}\right)$ may be obtained from the generating series $F_{\mathcal{E}_{n}}$ simply by replacing $x_{e}$, by $\mathbf{P}\left(e_{i}\right)$ and wrapping the vector $\Pi^{T}$ around the front and 1 around the back. We can formalize this concept by defining the mapping:

$$
\Delta: R \ll x_{0}, x_{1} \gg \longrightarrow M_{N}(R): x_{k} \mapsto \mathbf{P}(k),
$$

acting as a homomorphism to the whole of the ring. $M_{N}(R)$ is the ring of all $N \times N$ matrices with entries taken from $R$ (the field of real numbers). The probability of the set $\mathcal{E}_{n}$ may be expressed very compactly as:

$$
P\left(\mathcal{E}_{n}\right)=\boldsymbol{\Pi}^{T}\left(\Delta F_{\mathcal{E}_{n}}\right) \mathbf{1} .
$$

The main step to find $P\left(\mathcal{E}_{n}\right)$ is to determine the generating series $F_{\mathcal{E}_{n}}$. The key point to solve problems of this type is to find a bijection that expresses the set $\mathcal{E}_{n}$ in terms of concatenation products of binary strings. More examples will be addressed in the next section.

\section{DERIVING BURST STATISTICS FROM GENERATING SERIES}

In this section we will derive expressions for two important statistics:

- The error weight probability, $P(m, n)$, the probability of exactly $m$ errors occurring in a block of $n$ bits. This measure is important to determining the performance of block codes and interleaving on HMC models.
- The multigap distribution, $M(r, l)$, is the probability of $r$ consecutive gaps in a sequence of length $l$. The multigap distribution has been used as a test of non-renewalness of the error process.

In all cases, we first obtain an expression for an $N$-state HMC in terms of the matrices $\Pi, P(0), P(1)$. We now define some notation. If $s$ and $z$ are commutative indeterminates, $\left[s^{k} z^{n}\right] T(s, z)$ denotes the coefficient of $s^{k} z^{n}$ in the formal power series $T(s, z)$. If $\mathcal{A}$ is a set of sequences, $\mathcal{A}^{\star}$ is the set of all sequences formed by concatenating any number of sequences in $\mathcal{A}$. The identity matrix will be denoted by $\boldsymbol{I}$.

\subsection{The Error Weight Distribution $P(m, n)$}

We wish to determine the probability of the set $\mathcal{E}_{n}$ composed of sequences of Hamming weight $m$ and length $n$. The generating series for $\mathcal{E}_{n}$ is obtained directly from the generating series for the set of all binary sequences, $\{0,1\}^{\star}$, by defining the indeterminate $z$ to mark the length of the sequence and $s$ to mark the number of 1's. Since $F_{\{0,1\}^{*}}=\left(1-x_{0}-x_{1}\right)^{-1}$, it follows that $F_{\mathcal{E}_{n}}$ is:

$$
F_{\mathcal{E}_{n}}=\left[s^{m} z^{n}\right]\left(1-x_{0} z-x_{1} s z\right)^{-1} \in R \ll x_{0}, x_{1} \gg .
$$

From Equations (10) and (11) the error weight distribution $P(m, n)$ is given by:

$$
\begin{aligned}
\mathbf{P}(m, n) & =\left[s^{m} z^{n}\right] \boldsymbol{\Pi}^{T}(\boldsymbol{I}-\mathbf{P}(0) z-\mathbf{P}(1) s z)^{-1} \mathbf{1} ; \\
& =\left[s^{m} z^{n}\right] H_{P}(s, z),
\end{aligned}
$$

where

$$
\begin{aligned}
H_{P}(s, z) & =\sum_{m, n} P(m, n) s^{m} z^{n} \quad \in R[s][[z]] \\
& =\boldsymbol{\Pi}^{T}(\boldsymbol{I}-\mathbf{P}(0) z-\mathbf{P}(1) s z)^{-1} \mathbf{1}
\end{aligned}
$$

The generating series $H_{P}(s, z)$ is a polynomial in $s$. Then, $H_{P}(s, z)$ is a formal power series in $z$ with a coefficient ring $R[s]$. We now specialize the calculation of $P(m, n)$ for the Gilbert-Elliott channel. An expression for $H_{P}(s, z)$ can be obtained upon substitution of Equations (3)-(5) into (13). An explicit formula for $P(m, n)$ can be found by carrying out the partial fraction technique to extract the coefficient of Equation (12). Alternatively, it is simple go from generating series to recurrence formulas, which provides a rapid computational scheme for the problem. From Equation (12) we can prove that $P(m, n)$ for the Gilbert-Elliott channel satisfies the following 6-term recurrence formula:

$$
\begin{aligned}
& P(m, n)= \\
& -(Q(1-g)+q(1-b)-(2-g-b)) P(m, n-1) \\
& +(b(1-q)+g(1-Q)) P(m-1, n-1) \\
& -((1-b)(1-g)(1-q-Q)) P(m, n-2) \\
& -((1-q-Q)(b+g-2 g b)) P(m-1, n-2) \\
& -((1-q-Q) g b) P(m-2, n-2)
\end{aligned}
$$


for $m \geq 0, n \geq 2$, with initial conditions:

$$
\begin{aligned}
& P(m, n)=0, \text { for } m, n<0, m>n \\
& P(0,0)=1 ; \\
& P(0,1)=\frac{q}{q+Q}(1-g)+\frac{Q}{q+Q}(1-b) \\
& P(1,1)=\frac{q}{q+Q} g+\frac{Q}{q+Q} b .
\end{aligned}
$$

A new recurrence formula for $P(m, n)$ for the $(2,1)$-EFC Fritchman channel is given below:

$$
\begin{aligned}
& P(m, n)= \\
& \left(\lambda_{0}+\lambda_{1}\right) P(m, n-1)+ \\
& \left(1-p_{2.0}-p_{2.1}\right) P(m-1, n-1)- \\
& \lambda_{0} \lambda_{1} P(m, n-2)- \\
& \left(\left(\lambda_{0}+\lambda_{1}\right)-p_{2.0}\left(1+\lambda_{1}\right)-p_{2.1}\left(1+\lambda_{0}\right)\right) P(m-1, n-2) \\
& +\left(\lambda_{0} \lambda_{1}-p_{2.1} \lambda_{0}-p_{2.0} \lambda_{1}\right) P(m-1, n-3)
\end{aligned}
$$

for $m \geq 0, n \geq 3$, with initial conditions:

$$
\begin{aligned}
& P(0,0)=1 \\
& P(0,1)=P(0) \\
& P(1,1)=P(1) \\
& P(0,2)=P(00) \\
& P(1,2)=2 P(01) \\
& P(2,2)=P(11)
\end{aligned}
$$

Let the random variable $E^{n}$ be the number of errors in a block of length $n$. It is obvious that $P\left(E^{n}=m\right)=P(m, n)$. Moments of the random variable $E^{n}$ of any order, $\mathbf{E}\left\{\left(E^{n}\right)^{k}\right\}$, can be obtained from the $k^{t / 2}$ derivative of the matrix ( $I-$ $\mathbf{P}(0) z-\mathbf{P}(1) s z)^{-1}$, since:

$$
\begin{aligned}
& \mathbf{E}\left\{E^{\prime \prime}\left(E^{n}-1\right) \ldots\left(E^{n}-k+1\right)\right\}= \\
& =\left[z^{\prime \prime}\right]\left\{\frac{\partial^{k}}{\partial s^{k}} H_{P}(s, \boldsymbol{z})\right\}_{s=1} \\
& =\left[\boldsymbol{z}^{n}\right] \boldsymbol{\Pi}^{T}\left\{\frac{\partial^{k}}{\partial s^{k}}(\boldsymbol{I}-\mathbf{P}(\mathbf{0}) \boldsymbol{z}-\mathbf{P}(\mathbf{1}) s z)^{-1}\right\}_{s=1} \mathbf{1} .
\end{aligned}
$$

An exact formula for $\frac{\partial^{h}}{\partial s^{h}}(I-\mathbf{P}(0) z-\mathbf{P}(1) s z)^{-1}$ will be stated without proof in the next lemma.

Lemma 4..1 The $k^{\text {th }}$ partial derivative of the matrix $\mathbf{A}(s, z) \triangleq(I-\mathbf{P}(0) z-\mathbf{P}(1) s z)^{-1}$ is:

$$
\frac{\partial^{k}}{\partial s^{k}} \boldsymbol{A}(s, z):=: k !:(\boldsymbol{A}(s, z) P(1) z)^{k}: \mathbf{A}(s, z) \text {. }
$$

Using the result of the lemma, we are able to prove the following result:

$$
\begin{aligned}
& \mathbf{E}\left\{E^{n}\left(E^{n}-1\right) \ldots\left(E^{n}-k+1\right)\right\}= \\
& =k !\left[z^{n}\right] \boldsymbol{\Pi}^{T}\left((\boldsymbol{I}-\mathbf{P} z)^{-1} \mathbf{P}(1) z\right)^{k}(\boldsymbol{I}-\mathbf{P} z)^{-1} \mathbf{1} \\
& =k !\left[z^{n}\right] \boldsymbol{\Pi}^{T}\left((\boldsymbol{I}-\mathbf{P} z)^{-1} \mathbf{P}(\mathbf{1}) z\right)^{k} \frac{\mathbf{1}}{1-z} \\
& =k !\left[z^{n-k}\right] \boldsymbol{\Pi}^{T}\left((\boldsymbol{I}-\mathbf{P} z)^{-1} \mathbf{P}(1)\right)^{k} \frac{\mathbf{1}}{1-i} \\
& =k ! \sum_{j=0}^{n-k}\left[z^{j}\right] \boldsymbol{\Pi}^{T}\left((\boldsymbol{I}-\mathbf{P} z)^{-1} \mathbf{P}(1)\right)^{k} \mathbf{1}
\end{aligned}
$$

It is easy to see from Equation (14) that $\mathbf{E}\left\{E^{n}\right\}=n P(1)$ where $P(1)$ is defined in Equation (6). Moreover, the variance of $E^{\prime \prime}$, for a general HMC model can be found directly from (14) by setting $n=2$. The final expression is:

$$
\begin{aligned}
\operatorname{Var}\left(E^{n}\right) & =\mathbf{E}\left\{\left(E^{n}\right)^{2}\right\}-(n p(1))^{2} \\
& =2 \sum_{j=0}^{n-2}(n-j-1) \Pi^{T} \mathbf{P}(1) \mathbf{P}^{j} \mathbf{P}(1) \mathbf{1} \\
& +n P(1)(1-n P(1))
\end{aligned}
$$

From the variance of $E^{n}$, we can readily calculate the correlation coefficient between two blocks of length $n$. Consider a block of length $k n$ formed as a concatenation of $k$ blocks of length $n$. Let the random variable $E_{k}^{n}$ be the number of errors of the $k^{\text {th }}$ block. The correlation coefficient between $E_{0}^{n}$ and $E_{k}^{n}$ is given by [3]:

$$
\rho_{k}^{n}=\frac{\operatorname{Var}\left(E^{(k-1) n}\right)-2 \operatorname{Var}\left(E^{k n}\right)+\operatorname{Var}\left(E^{(k+1) n}\right)}{2 \operatorname{Var}\left(E^{n}\right)} .
$$

The quantity $\rho_{k}^{n}$ can be a useful indicator to select codes. For example, as mentioned in reference [3], the burst-trapping code works well for channels whose $\rho_{k}^{n}$. is negative. It is easy to show that for the Gilbert-Elliott channel, $\rho_{k}^{\prime \prime}$ is positive for all values of $k$ and $n$, as long as $0<(1-q-Q)<1$. A positive correlation indicates that whenever a block $i$ has more than the average number of errors, block $i+k$ has the tendency to have more than average.

\subsection{The Multigap Distribution $M(r, l)$}

The length of a gap is the number of zeros between two errors plus one (the last error is included). The error process $\left\{E_{k}\right\}_{k=1}^{\infty}$ can be regarded as a sequence of gaps $\left\{G_{k}\right\}_{k=1}^{\infty}$, where $G_{k}$ is the length of the $k^{t h}$ gap. The gap process is a convenient representation for the error sequence, since a large number of consecutive 0 's is expected to occur on channels with low bit error probability. Let the random variable $G^{r}=\sum_{i=k}^{k+r-1} G_{k}$ be the sum of $r$ consecutive gap lengths $G_{k}$. The multigap length distribution, denoted as $M(r, l)$, is defined as $M(r, l)=P\left(G^{r}=l\right)$. If the error process were renewal, this means that $\left\{G_{k}\right\}_{i=1}^{\infty}$ are independent random variables, then the variance of $G^{r}$ is $\operatorname{Var}\left(G^{r}\right)=r \operatorname{Var}\left(G_{1}\right)$.

The problem of finding $M(r, l)$ may be formulated as follows: Find the probability of the set $\mathcal{E}_{l}$, composed of binary sequences of length $l$ such that the $r^{t h}$ error will occur at the $l^{\text {th }}$ time interval. Note that the set of all sequences that ends with a 1 may be expressed as $\left\{0^{\star} 1\right\}^{\star}$. Let the indeterminate $z$ mark the length of the sequence and let $s$ mark the occurrence of a 1 . The generating series for the set $\mathcal{E}_{l}$ may be obtained from $\left\{0^{\star} 1\right\}^{\star}$ by replacing:

$$
\begin{array}{lll}
0^{\star} & \text { by } 1+x_{0} z+x_{0}^{2} z^{2}+\ldots=\left(1-x_{0} z\right)^{-1} \\
1 & \text { by }
\end{array}
$$

It follows that $F_{\mathcal{E}_{1}}$ is:

$$
F_{\mathcal{C}_{1}}=\left[s^{r} z^{l}\right]\left(1-\left(1-x_{0} z\right)^{-1} x_{1} s z\right)^{-1} \in R \ll x_{0}, x_{1} \gg \text {. }
$$

The multigap distribution $M(r, l)$ is the probability of the set $\mathcal{E}_{l}$, conditioned on $E_{0}=1$. Then

$$
M(r, l)=\left[s^{r} z^{l}\right] H_{M}(s, z),
$$


where the generating series $H_{M}(s, z)$ is:

$$
\begin{aligned}
& H_{M}(s, z)=\sum_{r, l} M(r, l) s^{r} z^{l} \quad \in R[s][[z]] ; \\
& =\frac{1}{P(1)} \mathbf{\Pi}^{T} \mathbf{P}(\mathbf{1})\left(\boldsymbol{I}-(\boldsymbol{I}-\mathbf{P}(0) z)^{-1} \mathbf{P}(1) s z\right)^{-1} \mathbf{1}
\end{aligned}
$$

It is interesting to notice that the computational effort to calculate $M(r, l)$ and $P(m, n)$ is very closely related, as will be stated in the following proposition.

Proposition 4..1 For a general HMC model, the statistics $P(m, n)$ and $M(r, l)$ follow the same recurrence relation, but with different initial conditions.

Proof. The generating series $H_{P}(s, z)$ and $H_{M}(s, z)$ are the ratio of two polynomials in $s$ and $z$. The denominator polynomial is responsible for the recurrence relation, and the numerator polynomial defines the initial conditions. So, it suffices to prove that $H_{P}(s, z)$ and $H_{M}(s, z)$ have the same denominator polynomial. $H_{P}(s, z)$ is defined in Equation (13), and it follows from Cramer's rule that its denominator polynomial is $\operatorname{det}(\boldsymbol{I}-\mathbf{P}(0) z-\mathbf{P}(1) s z)$. Using the same argument, we can conclude from Equation (18) that the denominator polynomial of $H_{A I}(s, z)$ is the numerator of $\operatorname{det}\left(\boldsymbol{I}-(\boldsymbol{I}-\mathbf{P}(0) z)^{-1} \mathbf{P}(\mathbf{1}) s z\right)$. But:

$$
\begin{aligned}
& \operatorname{det}\left(\boldsymbol{I}-(\boldsymbol{I}-\mathbf{P}(0) z)^{-1} \mathbf{P}(1) s z\right)= \\
& =\operatorname{det}\left((\boldsymbol{I}-\mathbf{P}(0) z)^{-1}[(\boldsymbol{I}-\mathbf{P}(0) z)-\mathbf{P}(1) s z]:\right) ; \\
& =\operatorname{det}\left((\boldsymbol{I}-\mathbf{P}(0) z)^{-1}\right) \operatorname{det}((\boldsymbol{I}-\mathbf{P}(0) z)-\mathbf{P}(1) s z) ; \\
& =\frac{\operatorname{det}(\boldsymbol{I}-\mathbf{P}(0) z-\mathbf{P}(1) s z)}{\operatorname{det}(\boldsymbol{I}-\mathbf{P}(0) z)} .
\end{aligned}
$$

Therefore, the proof is complete. as:

The variance of $G^{r}$, denoted as $\operatorname{Var}\left(G^{r}\right)$, can be expressed

$$
\operatorname{Var}\left(G^{r}\right)=\left[s^{r}\right]\left\{\frac{\partial^{2} H_{\Lambda I}(s, z)}{\partial z^{2}}\right\}_{z=1}+\mathbf{E}\left\{G^{r}\right\}\left(1-\mathbf{E}\left\{G^{r}\right\}\right) .
$$

Adoul [3] defined a quantity called variation coefficient $K(r)$ for a channel as:

$$
\operatorname{Var}\left(G^{r}\right)=K(r) \operatorname{Var}_{B S C}\left(G^{r}\right),
$$

where $\operatorname{Var}_{B S C}\left(G^{r}\right)=r(1-P(1)) / P(1)^{2}$ is the variance of $G^{r}$ for the BSC channel with crossover probability $P(1)$. Adoul [3] shows experimental curves for $K(r)$, as a function of $r$, for troposcatter channels. This curve was used to compare the spread of multigap lengths for a particular channel and its corresponding BSC. It is important to notice that for renewal processes $K(r)=K(1)$, for all $r$, that is, the curve $K(r)$ versus $r$ is a constant for all $r$. An expression for $K(r)$ for the renewal $(2,1)$-EFC model is given below:

$$
\begin{aligned}
K(r)= & \left(1+\lambda_{0}\right)\left(1+\lambda_{1}\right)\left(p_{2,1}\left(1-\lambda_{0}\right)+\right. \\
& \left.\left(1-\lambda_{1}\right) p_{2,0}\right)-\left(\left(1-\lambda_{0}\right) p_{2,1}+\right. \\
& \left.\left(1-\lambda_{1}\right) p_{2,0}\right)^{2} /\left(\left(1-\lambda_{0}\right)\left(1-\lambda_{1}\right)\right. \\
& \left.+p_{2,0}\left(1-\lambda_{1}\right)+p_{2,1}\left(1-\lambda_{0}\right)\right) \\
& \left(p_{2,0}\left(1-\lambda_{1}\right)+p_{2,1}\left(1-\lambda_{0}\right)\right)
\end{aligned}
$$

Table 1: $K(1)$ versus $d$

\begin{tabular}{|c|c|}
\hline \hline$d$ & $K(1)$ \\
\hline 1 & 4.443 \\
2 & 2.761 \\
3 & 2.100 \\
4 & 1.790 \\
5 & 1.600 \\
10 & 1.202 \\
20 & 1.061 \\
\hline
\end{tabular}

Since $K(r)$ is constant with $r$, the process is renewal. Let us sample the process $e_{n}$ to obtain the process $z_{n}$, or $z_{n}=e_{d n}$, for $d=1,2, \cdots$. Using the above theory, it can be shown that $z_{n}$ is a renewal process. Table 1 shows the values of $K(1)$ versus $d$ for the sampled process $z_{n}$ when the HMC model is the $(2,1)$-EFC channel given by Equation (8).

Adoul [3] defined a process whose $K(1)$ is greater than one as a more variable process, in the sense that the gap lengths spread widely from their mean value. In this case, errors have a trend to be clustered. The further $K(1)$ is from 1, the more pronounced is this trend. As the value of $d$ increases, the process $z_{n}$ tends to become memoryless and $K(1)$ tends to 1. If we encompass the channel with an interleaving and an deinterleaving with finite interleaving depth $d$, we can regard the sampled process $z_{n}$ as the error sequence at each row of the deinterleaver. We can use the table above to investigate the minimum value of $d$ that renders the channel memoryless.

Figure 2 illustrates the behavior of $K(r)$ for Gilbert-Elliott channels for two different sets of parameters. The models denoted as $\mathrm{M} 1$ and $\mathrm{M} 2$ in the figure have parameters $\left(Q=0.0022, q=0.11, b=0.4, g=10^{-2}\right)$ and $(Q=$ $4 \times 10^{-6}, q=4.7 \times 10^{-4}, b=0.3, g=10^{-3}$ ), respectively. The plots show that Gilbert-Elliott channels are able to model channels with gap lengths that spread widely from their mean value.

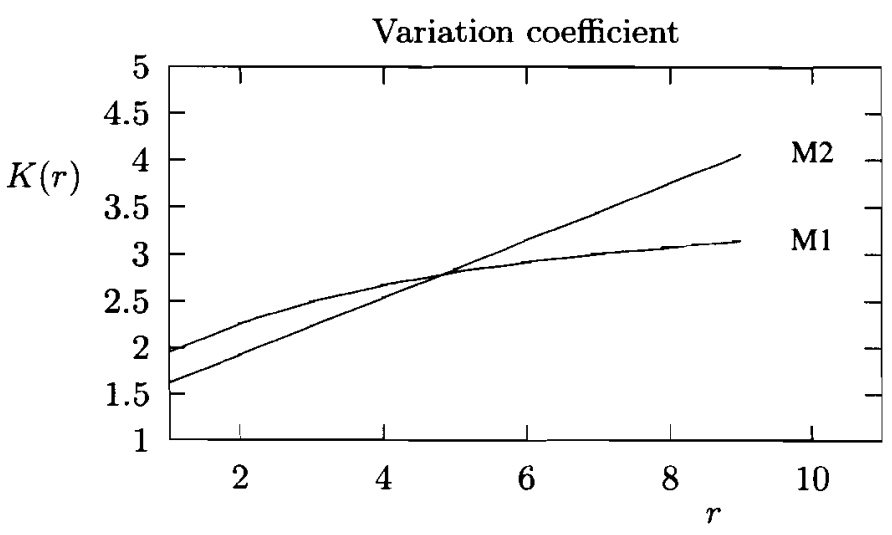

Figure 2: $K(r)$ as a function of $r$, for Gilbert-Elliott channels. 


\section{CONCLUSIONS}

A combinatorial approach has been presented to derive various statistics of interest in the analysis of general HMC models. The method has two main steps. First, the set of all permissible sequences that constitute the error event is enumerated. Second, a linear mapping incorporates the parameters of the model into the generating series. The specific new results developed in this paper are stressed below: (i) a new recurrence formula for $P(m, n)$ for the Gilbert-Elliott channel and for the (2,1)-EFC model; (ii) an expression for the variance of the multigap distribution; (iii) the fact that the multigap and the error weight distribution follows the same recurrence formulas. It is clear that many other problems of significance in the evaluation of communication system performance will also be amenable to the application of these techniques.

\section{REFERENCES}

[1] L. N. Kanal and A.R.K. Sastry, "Models for channels with memory and their applications to error control," Proceedings of the IEEE, Vol. 66, pp. 724-744, July 1978.

[2] E. O. Elliott, "Estimates of error rates for codes on burstnoise channels," The Bell System Technical Journal, Vol. 42, pp. 1977-1997, September 1963.

[3] J. A. Adoul, "Error intervals and cluster density in channel modeling," IEEE Transactions on Information Theory, vol.20, pp. 125-129, January 1974.

[4] I. P. Goulden and D. M. Jackson, Combinatorial Enumeration, John Wiley \& Sons, 1983.

[5] B. D. Fritchman, "A binary channel characterization using partitioned Markov chains," IEEE Transactions on Information Theory, Vol. 13, pp. 221-227, April 1967.

Cecílio Pimentel For biographical information please see page 107 of this issue. 